\title{
A CENTRAL LIMIT THEOREM FOR INTEGER PARTITIONS
}

\author{
MANFRED MADRITSCH AND STEPHAN WAGNER
}

\begin{abstract}
Recently, Hwang proved a central limit theorem for restricted $\Lambda$-partitions, where $\Lambda$ can be any nondecreasing sequence of integers tending to infinity that satisfies certain technical conditions. In particular, one of these conditions is that the associated Dirichlet series has only a single pole on the abscissa of convergence. In the present paper, we show that this condition can be relaxed, and provide some natural examples that arise from the study of integers with restrictions on their digital (base- $b$ ) expansion.
\end{abstract}

\section{INTRODUCTION}

For a nondecreasing sequence $\Lambda=\left(\Lambda_{1}, \Lambda_{2}, \ldots\right)$ of positive integers with $\Lambda_{k} \rightarrow \infty$, a restricted $\Lambda$-partition of $n$ is a subsequence of $\Lambda$ that sums to $n$, i.e.,

$$
\sum_{j=1}^{s} \Lambda_{i_{j}}=n
$$

with $i_{1}<i_{2}<\ldots<i_{s}$. On the other hand, if repetitions are allowed (i.e. $i_{1} \leq i_{2} \leq \ldots \leq i_{s}$ ), one speaks of unrestricted $\Lambda$-partitions. The number of restricted/unrestricted $\Lambda$-partitions is denoted by $q_{\Lambda}(n)$ and $p_{\Lambda}(n)$, respectively. There is a wealth of literature on the enumeration of restricted or unrestricted $\Lambda$-partitions, see for instance [2] and the references therein. Ingham [13] provides a Tauberian theorem that results in an asymptotic formula for the number of $\Lambda$-partitions under certain technical conditions. This approach was somewhat improved by Meinardus [15], who was able to remove a monotonicity condition necessary in Ingham's approach. Essentially, if the Dirichlet generating function of $\Lambda$ has a simple pole at $\alpha>0$ and can be analytically continued into a half-plane $\operatorname{Re} s \geq-\alpha_{0}$ with $\alpha_{0}>0$, and some other (fairly mild) conditions are satisfied, one obtains an asymptotic formula of the type

$$
p_{\Lambda}(n) \sim A \cdot n^{\kappa} \exp \left(B \cdot n^{\alpha /(\alpha+1)}\right)
$$

for unrestricted $\Lambda$-partitions. Under more general conditions, Roth and Szekeres [16] were able to prove slightly weaker theorems for both restricted and unrestricted partitions. The following holds for restricted partitions, the theorem for unrestricted partitions is similar:

Theorem 1 ([16, Theorem 2]). Assume that the following conditions hold:

- $\alpha^{-1}=\lim _{k \rightarrow \infty} \frac{\log \Lambda_{k}}{\log k}$ exists,

- $J_{k}=\inf \left\{(\log k)^{-1} \sum_{\nu=1}^{k}\left\|\Lambda_{\nu} \alpha\right\|^{2}\right\} \rightarrow \infty$ as $k \rightarrow \infty$, where the infimum is taken over all $\beta$ with $\frac{1}{2} \Lambda_{k}^{-1}<\beta \leq \frac{1}{2}$.

Date: December 23, 2008.

2000 Mathematics Subject Classification. 11P82; 11A63.

Key words and phrases. $\Lambda$-partitions, digital restrictions, central limit theorem. 
Then we have an asymptotic formula for $q_{\Lambda}(n)$, namely

$$
\begin{aligned}
q_{\Lambda}(n)= & \left(2 \pi A_{2}\right)^{-1 / 2} \exp \left(\sum_{k=1}^{\infty}\left(\frac{\eta \Lambda_{k}}{e^{\eta \Lambda_{k}}+1}+\log \left(1+e^{-\eta \Lambda_{k}}\right)\right)\right) \\
& \cdot\left(1+\sum_{\rho=1}^{m-2} D_{\rho}+O\left(n^{-(m-1) /(\alpha+1)+\delta}\right)\right),
\end{aligned}
$$

where $\eta=\eta(n)$ is determined from

$$
n=\sum_{k=1}^{\infty} \frac{\Lambda_{k}}{e^{\eta \Lambda_{k}}+1}
$$

and $D_{\rho}(\rho=1,2, \ldots)$ is given by

$$
D_{\rho}=A_{2}^{-6 \rho} \sum_{j_{1}=2}^{\infty} \ldots \sum_{j_{5 \rho}=2}^{\infty} c_{j_{1}, j_{2}, \ldots, j_{5 \rho}} A_{j_{1}} A_{j_{2}} \ldots A_{j_{5 \rho}},
$$

where the summation is subject to the condition

$$
j_{1}+\ldots+j_{5 \rho}=12 \rho,
$$

the c's are numerical coefficients and

$$
A_{j}=A_{j}(n)=\sum_{k=1}^{\infty} \Lambda_{k}^{j} g_{j}\left(e^{\eta \Lambda_{k}}\right)\left(e^{\eta \Lambda_{k}}+1\right)^{-j} .
$$

Here, $g_{j}$ is a polynomial of degree $<j$. In particular, $g_{1}(x)=1$ and $g_{2}(x)=x$.

The length (number of summands) of a partition is one of the most natural parameters to study. For unrestricted partitions, the distribution of the number of summands is typically not Gaussian, which is rather rare in combinatorial problems of similar type. Indeed, it was shown by Erdős and Lehner [4] that the number of summands asymptotically follows an extreme-value distribution in the special case $\Lambda=\mathbb{N}$, a result that was further extended in many directions, see for instance [7]. Similar results are known for the distribution of distinct elements in unrestricted partitions, see [11, 17]. On the other hand, the limit distribution is actually Gaussian if restricted partitions are considered, as was shown by Hwang [12, who extended a previous result of Erdös and Lehner. His conditions are essentially taken from the aforementioned paper of Meinardus [15]. Specifically, Hwang's central limit theorem reads as follows:

Theorem 2 ([12, Theorem 1]). Suppose that the sequence $\Lambda$ satisfies the following conditions:

(M1) The Dirichlet series $D(s)=\sum_{k \geq 1} \Lambda_{k}^{-s}$ converges in the half-plane $\operatorname{Re} s>\alpha>0$, and can be analytically continued into the half-plane $\operatorname{Re} s \geq-\alpha_{0}$ for some $\alpha_{0}>0$. In $\operatorname{Re} s \geq-\alpha_{0}$, $D(s)$ is analytic except for a simple pole at $s=\alpha$ with residue $A$.

(M2) There exists an absolute constant $c_{1}$ such that $D(s) \ll|t|^{c_{1}}$ uniformly for $\operatorname{Re} s \geq-\alpha_{0}$ as $|t| \rightarrow \infty$.

(M3) Define $g(\tau)=\sum_{k \geq 1} e^{-\Lambda_{k} \tau}$, where $\tau=r+i y$ with $r>0$ and $-\pi \leq y \leq \pi$. There exists a positive constant $c_{2}$ such that $g(r)-\operatorname{Re} g(\tau) \geq c_{2}(\log (1 / r))^{2+4 / \alpha^{2}}$ uniformly for $\pi / 2 \leq|y| \leq \pi$ as $r \rightarrow 0^{+}$.

Let $\varpi_{n}$ be the random variable counting the number of summands in a random restricted $\Lambda$ partition of $n$. Set $\kappa=A \Gamma(\alpha)\left(1-2^{-\alpha}\right) \zeta(\alpha+1)$, where $\Gamma$ and $\zeta$ are the Gamma function and the Riemann zeta function, respectively. Furthermore, set

$$
\begin{aligned}
\mu_{n} & =(\kappa \alpha)^{1 /(\alpha+1)} \frac{\left(1-2^{1-\alpha}\right) \zeta(\alpha)}{\alpha\left(1-2^{-\alpha}\right) \zeta(\alpha+1)} n^{\alpha /(\alpha+1)} \\
\sigma_{n}^{2} & =(\kappa \alpha)^{1 /(\alpha+1)}\left(\frac{\left(1-2^{2-\alpha}\right) \zeta(\alpha-1)}{\alpha\left(1-2^{-\alpha}\right) \zeta(\alpha+1)}-\frac{\left(1-2^{1-\alpha}\right)^{2} \zeta(\alpha)^{2}}{(\alpha+1)\left(1-2^{-\alpha}\right)^{2} \zeta(\alpha+1)^{2}}\right) n^{\alpha /(\alpha+1)} .
\end{aligned}
$$


Then $\varpi_{n}$ is asymptotically normally distributed with mean $\mathbb{E}\left(\varpi_{n}\right) \sim \mu_{n}$ and variance $\mathbb{V}\left(\varpi_{n}\right) \sim \sigma_{n}^{2}$ :

$$
\mathbb{P}\left(\frac{\varpi_{n}-\mu_{n}}{\sigma_{n}}<x\right)=\frac{1}{\sqrt{2 \pi}} \int_{-\infty}^{x} e^{-t^{2} / 2} \mathrm{~d} t+o(1),
$$

uniformly for all $x$ as $n \rightarrow \infty$.

It is generally required in all the mentioned results that Meinardus' condition on the Dirichlet generating function (analyticity on a half-plane except for a simple pole at $\alpha$ ) is satisfied. The examples given by Hwang include, for instance, powers $\left(\Lambda_{j}=j^{\ell}\right)$ or arithmetic progressions $\left(\Lambda_{j}=a+b j\right.$, where $a$ and $b$ are coprime). However, there are fairly natural integer sequences which do not satisfy this condition. Specifically, we consider integers satisfying certain conditions on their digits in base $b$; various authors, most notably Gel'fond [10], studied arithmetical properties of integers defined by such conditions. A typical example are numbers with missing digits, which were treated, among others, by Erdős, Mauduit and Sárközy [5, 6]: if, for instance, one considers only those integers which do not contain the digit 2 in their base- 3 expansion, one obtains the sequence

$$
\Lambda=(1,3,4,9,10,12,13,27, \ldots),
$$

which is Sloane's A005836 [18. It is not difficult to see (and will be shown later) that the corresponding Dirichlet generating function doesn't only have a pole at $\alpha=\frac{\log 2}{\log 3}$, but also further poles along the line $\operatorname{Re} s=\alpha$. This and similar examples form our motivation for replacing Hwang's conditions (M1) and (M2) by slightly weaker assumptions that allow for further poles with nonnegative real part. It will turn out that the limit distribution is still Gaussian under these assumptions and thus specifically for numbers with missing digits.

In order to get a flavor of the new phenomena that occur, let us apply the result of Roth and Szekeres (Theorem 1) to the sequence of integers with missing digits. Let $b>2$ be an integer, and let $D \subseteq\{0,1, \ldots, b-1\}$ be a set of digits $(1<|D|<b)$. Without loss of generality, we will always assume that the digits in $D$ don't have a common divisor (otherwise, simply divide everything by the greatest common divisor). Let $\mathcal{M D}(b, D)$ be the set of positive integers with the property that all digits in the $b$-ary representation come from the set $D$, i.e.,

$$
\mathcal{M D}(b, D):=\left\{\sum_{i=0}^{k-1} a_{i} b^{i} \mid k \in \mathbb{N}, a_{i} \in D\right\} \backslash\{0\} .
$$

Now we would like to determine (asymptotically) the number of restricted $\mathcal{M D}(b, D)$-partitions. To this end, we need some information on the Dirichlet generating function of such a set, which is provided by the following lemma.

Lemma 3. Let $D(s)$ be defined by

$$
D(s)=\sum_{m \in \mathcal{M D}(b, D)} m^{-s} .
$$

Then we have

$$
D(s)=\left(1-|D| b^{-s}\right)^{-1} R(s),
$$

where $R$ is analytic within the right half-plane

$$
\left\{s \in \mathbb{C} \mid \operatorname{Re} s>\frac{\log |D|}{\log b}-1\right\}
$$

and satisfies $R(s) \ll|s|$ uniformly on

$$
\left\{s \in \mathbb{C} \mid \operatorname{Re} s \geq \frac{\log |D|}{\log b}-1+\varepsilon\right\} .
$$

Proof. Note that

$$
\mathcal{M D}(b, D)=\left\{b n_{0}+a_{0} \mid n_{0} \in \mathcal{M D}(b, D) \cup\{0\}, a_{0} \in D\right\} \backslash\{0\} .
$$


Therefore, we have

$$
\begin{aligned}
R(s) & =\left(1-|D| b^{-s}\right) D(s) \\
& =\sum_{n \in \mathcal{M} \mathcal{D}(b, D)} \sum_{a \in D}\left(\frac{1}{(b n+a)^{s}}-\frac{1}{(b n)^{s}}\right)+\sum_{a \in D \backslash\{0\}} \frac{1}{a^{s}} .
\end{aligned}
$$

So $R(s)$ is a Dirichlet series again, which means that it is analytic within a right half-plane. Hence, in order to prove the theorem, it is sufficient to show that the estimate $R(s) \ll|s|$ holds (uniformly) for $\sigma=\operatorname{Re} s \geq \frac{\log |D|}{\log b}-1+\varepsilon$. To this end, note that

$$
\sum_{a \in D}\left(\frac{1}{(b n)^{s}}-\frac{1}{(b n+a)^{s}}\right) \ll|s| n^{-\sigma-1},
$$

and this estimate holds uniformly as $\sigma$ is restricted to a compact set. Furthermore, $\mid \mathcal{M D}(b, D) \cap$ $\left.\left[b^{l-1}, b^{l}\right)|\leq| D\right|^{l}$, so that we obtain

$$
\begin{aligned}
\sum_{n \in \mathcal{M} \mathcal{D}(b, D)} \sum_{a \in D}\left(\frac{1}{(b n)^{s}}-\frac{1}{(b n+a)^{s}}\right) & \ll|s| \sum_{n \in \mathcal{M} \mathcal{D}(b, D)} n^{-\sigma-1} \\
& \leq|s| \sum_{l=0}^{\infty} \frac{|D|^{l}}{b^{(l-1)(\sigma+1)}} \\
& =|s| b^{\sigma+1} \frac{1}{1-|D| b^{-(\sigma+1)}}<\infty
\end{aligned}
$$

as long as $|D| b^{-(\sigma+1)}<1$ or $\sigma>\frac{\log |D|}{\log b}-1$, and the estimate is uniform for

$$
\sigma=\operatorname{Re} s \geq \frac{\log |D|}{\log b}-1+\varepsilon
$$

Thus, in order to obtain asymptotic estimates for sums of the type

$$
\sum_{m \in \mathcal{M D}(b, D)} f(m \eta)
$$

as given in Theorem 1, we can use the Mellin inversion formula together with Lemma 3, For a survey on Mellin transforms we refer the reader to [8, 9, 19]. We denote the Mellin transform of a function $h(x)$ by

$$
h^{*}(s)=\mathcal{M}[h(x) ; s]=\int_{0}^{\infty} h(x) x^{s-1} \mathrm{~d} s .
$$

First of all, we want to know the asymptotics of $\eta=\eta(n)$ in Theorem 1. Note that the Mellin transform of $f_{1}(x)=\frac{x}{e^{x}+1}$ is given by

$$
f_{1}^{*}(s)=\left(1-2^{-s}\right) \Gamma(s+1) \zeta(s+1),
$$

which is analytic for $\operatorname{Re} s>-1$. By the properties of the Mellin transform, we have

$$
\mathcal{M}\left[\sum_{m \in \mathcal{M} \mathcal{D}(b, D)} \frac{m x}{e^{m x}+1} ; s\right]=\left(1-2^{-s}\right) \Gamma(s+1) \zeta(s+1) D(s)
$$

Thus, shifting the path of integration in the Mellin inversion formula yields

$$
\sum_{m \in \mathcal{M D}(b, D)} \frac{m}{e^{\eta m}+1}=\eta^{-\alpha-1} U_{1}\left(\frac{\log \eta}{\log b}\right)+O\left(\eta^{-\alpha}\right),
$$

where $\alpha=\frac{\log |D|}{\log b}$ and $U_{1}$ is a 1-periodic function given by its Fourier series (for details, see Lemma 6 in Section 2)

$U_{1}(t)=\sum_{k \in \mathbb{Z}} \frac{1}{\log b}\left(1-2^{-\alpha+\frac{2 k \pi i}{\log b}}\right) \Gamma\left(1+\alpha-\frac{2 k \pi i}{\log b}\right) \zeta\left(1+\alpha-\frac{2 k \pi i}{\log b}\right) R\left(\alpha-\frac{2 k \pi i}{\log b}\right) \exp (2 k \pi i t)$. 
It follows that

$$
\eta=n^{-1 /(\alpha+1)} V\left(\frac{\log n}{\log |D|+\log b}\right)+O\left(n^{-2 /(\alpha+1)}\right),
$$

where $V$ is also 1-periodic. Similarly, the Mellin transform of $f_{2}(x)=\log \left(1+e^{-x}\right)$ is given by

$$
f_{2}^{*}(s)=\left(1-2^{-s}\right) \Gamma(s) \zeta(s+1),
$$

and we obtain

$$
\sum_{m \in \mathcal{M} \mathcal{D}(b, D)}\left(\frac{m \eta}{e^{m \eta}+1}+\log \left(1+e^{-\eta m}\right)\right)=\eta^{-\alpha} U_{2}\left(\frac{\log \eta}{\log b}\right)+C+O\left(\eta^{1-\alpha}\right)
$$

where $U_{2}$ is a 1-periodic function and $C$ a constant. Finally, the Mellin transform of $f_{3}(x)=\frac{x^{2} e^{x}}{\left(e^{x}+1\right)^{2}}$ is given by

$$
f_{3}^{*}(s)=\left(1-2^{-s}\right) \Gamma(s+2) \zeta(s+1),
$$

yielding an analogous asymptotic formula for $A_{2}$ in (1.1). Summing up, we obtain the following asymptotic formula:

Theorem 4. The number $q_{\mathcal{M D}}(n)$ of partitions into distinct elements of $\mathcal{M D}(b, D)$ is asymptotically

$$
\begin{aligned}
q_{\mathcal{M D}}(n)= & n^{-(\alpha+2) /(2 \alpha+2)} \exp \left(n^{\alpha /(\alpha+1)} W_{1}\left(\frac{\log \eta}{\log |D|+\log b}\right)\right) W_{2}\left(\frac{\log \eta}{\log |D|+\log b}\right) \\
& \cdot\left(1+O\left(n^{-\min (\alpha, 1-\alpha) /(\alpha+1)+\delta}\right)\right)
\end{aligned}
$$

for some 1-periodic functions $W_{1}, W_{2}$.

Given this asymptotic result for the number of partitions, it is natural to consider distributions as well. However, Hwang's Theorem (Theorem 2) is not directly applicable since there is not only a single pole on the abscissa of convergence of the relevant Dirichlet series, but rather a countable set of poles, as can be seen from Lemma 3. Therefore, we aim to extend Hwang's result in order to make it applicable to partitions into integers with missing digits and similar sequences of integers (see the examples in Section 4).

\section{Preliminaries and statement of the main Result}

In the following, we typically consider the case that the sequence $\Lambda$ is strictly increasing, i.e.

$$
\Lambda_{1}<\Lambda_{2}<\Lambda_{3}<\ldots,
$$

and so it will be convenient to write $\mathcal{S}$ for the set $\left\{\Lambda_{1}, \Lambda_{2}, \Lambda_{3}, \ldots\right\}$ (e.g. the set of integers with certain missing digits). However, everything extends to the case of multisets (i.e. the elements of $\mathcal{S}$ can be taken with multiplicity). In order to study the number of summands in partitions into distinct elements of $\mathcal{S}$, we define the bivariate generating function

$$
Q(u, z)=\prod_{m \in \mathcal{S}}\left(1+u z^{m}\right) .
$$

It is clear that the power of $u$ indicates the number of summands. For convenience, we mostly work with the logarithm of $Q$ and thus define the function

$$
f(u, \tau)=\log Q\left(u, e^{-\tau}\right)=\sum_{m \in \mathcal{S}} \log \left(1+u e^{-m \tau}\right) .
$$

We write $D(s)$ for the Dirichlet generating function of $\mathcal{S}$, i.e.,

$$
D(s)=\sum_{m \in \mathcal{S}} m^{-s}
$$

and we will use the notation

$$
\langle\alpha, \beta\rangle:=\{z \in \mathbb{C}: \alpha<\operatorname{Re} z<\beta\}
$$

for strips in the complex plane. Throughout this paper, we will assume that $\mathcal{S}$ satisfies the following conditions, which are slight modifications of Hwang's conditions (M1)-(M3): 
(M1') $D(s)$ converges in the half-plane $\operatorname{Re} s>\alpha>0$ and can be analytically continued to $\operatorname{Re} s \geq \alpha-\varepsilon$ with $\varepsilon>0$. On the line $\operatorname{Re} s=\alpha, D(s)$ has equidistant simple poles at $s=\alpha+2 \pi i k \omega$ with $k \in \mathbb{Z} ; A_{k}$ is the residue of $D(s)$ at $s=\alpha+2 \pi i k \omega$. Furthermore we assume that there are no further poles with $\operatorname{Re} s \geq \alpha-\varepsilon$.

(M2') There exists a sequence $T_{j} \rightarrow \infty$ and a positive constant $c_{1}$ such that

$$
D(s) \ll\left|T_{j}\right|^{c_{1}}
$$

uniformly for all $s \in\langle\alpha-\varepsilon, \alpha\rangle$ with $|\operatorname{Im} s|=T_{j}$. Furthermore we assume that $D$ satisfies

$$
D(\alpha-\varepsilon+i t) \ll|t|^{c_{1}} .
$$

(M3') Let $g(\tau)=\sum_{m \in \mathcal{S}} e^{-m \tau}$, where $\tau=r+i y$ with $r>0$ and $-\pi \leq y \leq \pi$. There exists a positive constant $c_{2}$ such that

$$
g(r)-\operatorname{Re} g(\tau) \geq c_{2}\left(\log \frac{1}{r}\right)^{2+4 / \alpha}
$$

uniformly for $\pi / 2 \leq|y| \leq \pi$ as $r \rightarrow 0^{+}$.

We will show that these conditions are sufficient for a central limit theorem as follows:

Theorem 5 (Main Theorem). Suppose that (M1')-(M3') hold. As in Theorem 2, let $\varpi_{n}$ be the number of summands of a random partition. Then $\varpi_{n}$ is asymptotically normally distributed with mean $\mathbb{E}\left(\varpi_{n}\right) \sim \mu_{n}$ and variance $\mathbb{V}\left(\varpi_{n}\right) \sim \sigma_{n}^{2}$ :

$$
\mathbb{P}\left(\frac{\varpi_{n}-\mu_{n}}{\sigma_{n}}<x\right)=\frac{1}{\sqrt{2 \pi}} \int_{-\infty}^{x} e^{-t^{2} / 2} \mathrm{~d} t+o(1),
$$

uniformly for all $x$ as $n \rightarrow \infty$. $\mu_{n}$ and $\sigma_{n}$ are defined as follows:

$$
\begin{aligned}
\mu_{n} & =\sum_{m \in \mathcal{S}} \frac{1}{e^{\eta m}+1}, \\
\sigma_{n}^{2} & =\sum_{m \in \mathcal{S}} \frac{e^{\eta m}}{\left(e^{\eta m}+1\right)^{2}}-\frac{\left(\sum_{m \in \mathcal{S}} \frac{m e^{\eta m}}{\left(e^{\eta m}+1\right)^{2}}\right)^{2}}{\sum_{m \in \mathcal{S}} \frac{m^{2} e^{\eta m}}{\left(e^{\eta m}+1\right)^{2}}},
\end{aligned}
$$

and $\eta$ is implicitly given by

$$
n=\sum_{m \in \mathcal{S}} \frac{m}{e^{\eta m}+1} .
$$

$\mu_{n}$ and $\sigma_{n}$ satisfy the following asymptotic formulas:

$$
\begin{aligned}
& \mu_{n} \sim n^{\alpha /(1+\alpha)} \Psi_{\mu}\left(\frac{\omega \log n}{\alpha+1}\right), \\
& \sigma_{n}^{2} \sim n^{\alpha /(1+\alpha)} \Psi_{\sigma}\left(\frac{\omega \log n}{\alpha+1}\right),
\end{aligned}
$$

for certain 1-periodic functions $\Psi_{\mu}$ and $\Psi_{\sigma}$. Finally, we have the following exponential bounds for the tails:

$$
\mathbb{P}\left(\frac{\varpi_{n}-\mu_{n}}{\sigma_{n}} \geq x\right) \leq \begin{cases}e^{-x^{2} / 2}\left(1+O\left((\log n)^{-3}\right)\right) & \text { if } 0 \leq x \leq n^{\alpha /(6 \alpha+6)} / \log n \\ e^{-n^{\alpha /(6 \alpha+6)} x /(2 \log n)}\left(1+O\left((\log n)^{-3}\right)\right) & \text { if } x \geq n^{\alpha /(6 \alpha+6)} / \log n\end{cases}
$$

and the same inequalities for $\mathbb{P}\left(\frac{\varpi_{n}-\mu_{n}}{\sigma_{n}} \leq-x\right)$.

The proof makes use of the saddle point method that is applied to the generating function $Q(u, z)$ ( cf. [12, 16]). Note that the definition of $\eta$ is analogous to that in Theorem 1 as we will see from the proof, this is precisely the choice for the saddle point. Harmonic sums over all elements of $\mathcal{S}$ (as in the definitions of $\eta, \mu_{n}, \sigma_{n}^{2}$ ) will occur repeatedly, and so we will make frequent use of the following important lemma: 
Lemma 6 ([8, Theorem 4]). Let $f(x)$ be continuous in $(0, \infty)$ with Mellin transform $f^{*}(s)$ having a non-empty fundamental strip $\langle\alpha, \beta\rangle$.

Assume that $f^{*}(s)$ admits a meromorphic continuation to the strip $\langle\gamma, \beta\rangle$ for some $\gamma<\alpha$ with at most a countable set of poles $P$ there, and is analytic on $\operatorname{Re} s=\gamma$. Assume also that there exists a real number $\eta \in(\alpha, \beta)$ and a sequence of horizontal segments $|\operatorname{Im} s|=T_{j}$ with $T_{j} \rightarrow+\infty$ such that

$$
f^{*}(s)=\mathcal{O}\left(|s|^{-r}\right) \quad \text { with } r>1
$$

holds on these segments uniformly for $\gamma \leq \operatorname{Re} s \leq \eta$. If $f^{*}(s)$ admits the singular expansion

$$
f^{*}(s) \asymp \sum_{\xi \in P} \frac{A_{\xi}}{s-\xi}
$$

for $s \in\langle\gamma, \alpha\rangle$, then an asymptotic expansion of $f(x)$ at 0 is

$$
f(x)=\sum_{\xi \in P} A_{\xi} x^{-\xi}+\mathcal{O}\left(x^{-\gamma}\right) .
$$

In order to apply the Mellin calculus to the function $f$ defined in (2.2), we need the Mellin transform of $\log \left(1+u e^{-x}\right)$, which we denote by $Y(u, s)$ :

$$
Y(u, s)=\mathcal{M}\left[\log \left(1+u e^{-x}\right) ; s\right]=\int_{0}^{\infty} \log \left(1+u e^{-x}\right) x^{s-1} \mathrm{~d} x .
$$

The following lemma collects some of the important properties of $Y(u, s)$.

Lemma 7 ([12, Lemma 1]). For each fixed $u$ lying in the cut-plane $\mathbb{C} \backslash(-\infty,-1]$, the function $Y(u, s)$ can be meromorphically continued into the whole s-plane with simple poles at $s=$ $0,-1,-2, \ldots$ Moreover, $Y(u, s)$ satisfies the estimate

$$
|Y(u, \sigma+i t)| \ll e^{-(\pi / 2-\varepsilon)|t|} \text { for any } \varepsilon>0 \text { as }|t| \rightarrow+\infty
$$

uniformly as $\sigma$ and $u$ are restricted to compact sets.

By partial integration of $Y(u, s)$ we get

$$
Y(u, s)=\int_{0}^{\infty} \log \left(1+u e^{-x}\right) x^{s-1} \mathrm{~d} x=\frac{1}{s} \int_{0}^{\infty} \frac{1}{u^{-1} e^{x}+1} x^{s} \mathrm{~d} x
$$

and analogously

$$
\begin{aligned}
& Y(u, s)=\frac{1}{s(s+1)} \int_{0}^{\infty} \frac{u^{-1} e^{x}}{\left(u^{-1} e^{x}+1\right)^{2}} x^{s+1} \mathrm{~d} x \\
& Y(u, s)=\frac{1}{s(s+1)(s+2)} \int_{0}^{\infty} \frac{u^{-1} e^{x}\left(u^{-1} e^{x}-1\right)}{\left(u^{-1} e^{x}+1\right)^{3}} x^{s+2} \mathrm{~d} x
\end{aligned}
$$

\section{Proof of THE MAIN THEOREM}

In order to prove Theorem 5, we need an asymptotic formula for

$$
Q_{n}(u)=\left[z^{n}\right] Q(u, z) .
$$

Using Cauchy's residue theorem and the substitution $z=e^{-(r+i t)}$, this can be written as

$$
Q_{n}(u)=\frac{1}{2 \pi i} \oint_{|z|=e^{-r}} z^{-n-1} Q(u, z) \mathrm{d} z=\frac{e^{n r}}{2 \pi} \int_{-\pi}^{\pi} \exp (i n t+f(u, r+i t)) \mathrm{d} t
$$

for any $r>0$. Let $\delta>0$ be any fixed number in the unit interval. Throughout the proof, we assume that $\delta \leq u \leq \delta^{-1}$. Thus, "uniformly in $u$ " means "uniformly as $\delta \leq u \leq \delta^{-1}$ ". Now we apply the saddle-point method: in the following, $r=r(n, u)$ is chosen in such a way that

$$
\left.\frac{\partial(i n t+f(u, r+i t))}{\partial t}\right|_{t=0}=i n-i \sum_{m \in \mathcal{S}} \frac{m}{u^{-1} e^{r m}+1}=0 .
$$


or equivalently

$$
n=\sum_{m \in \mathcal{S}} \frac{m}{u^{-1} e^{r m}+1} .
$$

Note that the right hand side is strictly decreasing and thus bijective as a function of $r$. Therefore there is a unique $r$ that satisfies this equation. Furthermore, $r$ is strictly decreasing as a function of $n$ (and tends to 0 as $n \rightarrow \infty$ ) and strictly increasing as a function of $u$.

We can make use of Lemma 6 to find an asymptotic formula for the sum in the definition of $r$. Recall that the Mellin transform of $\frac{x}{u^{-1} e^{x}+1}$ is given by

$$
\mathcal{M}\left[\frac{x}{u^{-1} e^{x}+1} ; s\right]=s Y(u, s),
$$

and thus

$$
\mathcal{M}\left[\sum_{m \in \mathcal{S}} \frac{m x}{u^{-1} e^{x m}+1} ; s\right]=s Y(u, s) D(s) .
$$

So Lemma 6 yields

$$
\begin{aligned}
n & =\sum_{m \in \mathcal{S}} \frac{m}{u^{-1} e^{r m}+1}=\frac{1}{r} \sum_{m \in \mathcal{S}} \frac{r m}{u^{-1} e^{r m}+1} \\
& =\frac{1}{r} \sum_{j \in \mathbb{Z}} A_{j}(\alpha+2 \pi i j \omega) Y(u, \alpha+2 \pi i j \omega) r^{-(\alpha+2 \pi i j \omega)}+\mathcal{O}\left(r^{-(\alpha+1)+\varepsilon}\right) \\
& =r^{-(\alpha+1)} \sum_{j \in \mathbb{Z}} A_{j}(\alpha+2 \pi i j \omega) Y(u, \alpha+2 \pi i j \omega) \exp (-2 \pi i j \omega \log r)+\mathcal{O}\left(r^{-(\alpha+1)+\varepsilon}\right) \\
& =r^{-(\alpha+1)} \Phi_{1}(u, \omega \log r)+\mathcal{O}\left(r^{-(\alpha+1)+\varepsilon}\right)
\end{aligned}
$$

for a 1-periodic function $\Phi_{1}$ that is given by the Fourier series

$$
\Phi_{1}(u, v)=\sum_{j \in \mathbb{Z}} A_{j}(\alpha+2 \pi i j \omega) Y(u, \alpha+2 \pi i j \omega) \exp (-2 \pi i j v) .
$$

The properties of $Y$ summarized in Lemma 7 guarantee that this series is absolutely and uniformly convergent and infinitely differentiable. Also note that

$$
H(u, r)=\sum_{m \in \mathcal{S}} \frac{m}{u^{-1} e^{r m}+1}
$$

is a positive and monotonic function of $r$. Therefore, $\Phi_{1}(u, v)$ can never be 0 : otherwise, there are sequences $r_{1, k}$ and $r_{2, k}$ both tending to 0 such that

$$
H\left(u, r_{1, k}\right) \ll r_{1, k}^{-(\alpha+1)+\varepsilon} \text { and } H\left(u, r_{2, k}\right) \gg r_{2, k}^{-(\alpha+1)}
$$

(the latter simply follows from the fact that $\Phi_{1}$ is not identically 0 ), contradicting the monotonicity. Thus, $\Phi_{1}(u, v)$ must be bounded above and below by strictly positive constants (uniformly in $u$ ), which means that $r=\Theta\left(n^{-1 /(\alpha+1)}\right)$. More precisely, one has

$$
r \sim n^{-\frac{1}{\alpha+1}} \Psi_{1}\left(u, \frac{\omega \log n}{\alpha+1}\right)
$$

for a 1-periodic function $\Psi_{1}$, which will be used later.

For our application of the saddle point method, we need a uniform estimate as $t$ in the integral representation (3.1) is away from 0 . This is the main objective of the following two lemmas:

Lemma 8. For every integer $\ell \geq 0$ we have

$$
h(X)=\sum_{\substack{m \leq X \\ m \in \mathcal{S}}} m^{\ell} \gg X^{\alpha+\ell} .
$$


Proof. For a nonnegative integer $k$ we set

$$
G_{k}(X):=\sum_{\substack{m \leq X \\ m \in \mathcal{S}}} m^{\ell}\left(1-\frac{m}{X}\right)^{k} .
$$

Sums of this type can be written as integrals by means of the Mellin transform (see [9, Theorem $2.1])$ :

$$
G_{k}(X)=\frac{k !}{2 \pi i} \int_{c-i \infty}^{c+i \infty} \frac{D(s-\ell) X^{s}}{s(s+1) \ldots(s+k)} \mathrm{d} s
$$

for any $c>\alpha+\ell$. We choose $k$ large enough $\left(k>c_{1}\right.$, where the constant $c_{1}$ is taken as in (M2')) so as to make the resulting integral converge and shift the line of integration to the left (collecting residues at $s=\alpha+\ell+2 \pi i j \omega$ for every $j \in \mathbb{Z}$ ) to obtain

$$
\begin{aligned}
G_{k}(X)= & k ! \sum_{j \in \mathbb{Z}} \frac{A_{j} X^{\alpha+\ell+2 \pi i j \omega}}{(\alpha+\ell+2 \pi i j \omega)(\alpha+\ell+1+2 \pi i j \omega) \ldots(\alpha+\ell+k+2 \pi i j \omega)} \\
& +\frac{k !}{2 \pi i} \int_{\alpha+\ell-\varepsilon-i \infty}^{\alpha+\ell-\varepsilon+i \infty} \frac{D(s-\ell) X^{s}}{s(s+1) \ldots(s+k)} \mathrm{d} s \\
= & X^{\alpha+\ell} \Xi_{k}(\omega \log X)+\mathcal{O}\left(X^{\alpha+\ell-\varepsilon}\right),
\end{aligned}
$$

where $\Xi_{k}$ is a function of period 1, given by its Fourier series. Here, we made use of the fact that the integral can be estimated as follows:

$$
\begin{aligned}
\left|\int_{\alpha+\ell-\varepsilon-i \infty}^{\alpha+\ell-\varepsilon+i \infty} \frac{D(s-\ell) X^{s}}{s(s+1) \ldots(s+k)} \mathrm{d} s\right| & \leq X^{\alpha+\ell-\varepsilon} \int_{\alpha+\ell-\varepsilon-i \infty}^{\alpha+\ell-\varepsilon+i \infty}\left|\frac{D(s-\ell)}{s(s+1) \ldots(s+k)}\right| \mathrm{d} s \\
& \ll X^{\alpha+\ell-\varepsilon} \int_{\alpha+\ell-\varepsilon-i \infty}^{\alpha+\ell-\varepsilon+i \infty}|s|^{c_{1}-k-1} \mathrm{~d} s \\
& \ll X^{\alpha+\ell-\varepsilon} .
\end{aligned}
$$

Since $G_{k}(X)$ is nonnegative for all $X, \Xi_{k}(\omega \log X)$ must be nonnegative for all $X$. Now assume that $\Xi_{k}(\omega \log X)$ is 0 for some $X$. Equivalently,

$$
G_{k}\left(X_{n}\right) X_{n}^{-(\alpha+\ell)} \rightarrow 0
$$

for $X_{n}=X e^{n / \omega}$. Note that

$$
G_{k}(X) \leq h(X) \leq G_{k}(\beta X)\left(1-\frac{1}{\beta}\right)^{-k} .
$$

for every $k$ and $\beta>1$. Therefore, we also have

$$
G_{k}\left(X_{n} / \beta\right) X_{n}^{-(\alpha+\ell)} \rightarrow 0
$$

for any $\beta>1$, implying $\Xi_{k}(\omega(\log X-\log \beta))=0$. But then, $\Xi_{k}$ is identically 0 , an obvious contradiction. Hence, $\Xi_{k}$ is bounded above and below by strictly positive constants. Now, the left hand side inequality in (3.2) shows that $h(X) \gg X^{\alpha+\ell}$, as claimed.

The following simple corollary will be needed later:

Corollary 9. There is a constant $C>1$ such that the cardinality of $\mathcal{S} \cap(X, C X]$ satisfies

$$
|\mathcal{S} \cap(X, C X]| \gg X^{\alpha} \text {. }
$$

Proof. Set $\ell=0$ in the lemma; the proof shows that $h(X) \ll X^{\alpha}$ holds as well as $h(X) \gg X^{\alpha}$. Hence,

$$
|\mathcal{S} \cap(X, C X]|=h(C X)-h(X) \gg X^{\alpha}
$$

for sufficiently large $C$. 
Lemma 10. For any constant $c_{3}$ with $0<c_{3}<\alpha / 2$, there is a constant $c_{4}$ such that

$$
\frac{\left|Q\left(u, e^{-(r+i y)}\right)\right|}{Q\left(u, e^{-r}\right)} \leq \exp \left(-\frac{c_{4} u}{(1+u)^{2}}\left(\log \frac{1}{r}\right)^{2}\right)
$$

for $r^{1+c_{3}} \leq|y| \leq \pi$ as $r \rightarrow 0^{+}$.

Proof. We start by rewriting the quotient under consideration.

$$
\begin{aligned}
\left(\frac{\left|Q\left(u, e^{-(r+i y)}\right)\right|}{Q\left(u, e^{-r}\right)}\right)^{2} & =\prod_{m \in \mathcal{S}}\left(1-\frac{2 u e^{-m r}(1-\cos m y)}{\left(1+u e^{-m r}\right)^{2}}\right) \\
& \leq \exp \left(-\frac{2 u}{(1+u)^{2}} \sum_{m \in \mathcal{S}} e^{-m r}(1-\cos m y)\right)
\end{aligned}
$$

Using the definition of $g$ in (M3') we set

$$
G(r, y):=g(r)-\operatorname{Re} g(r+i y)=\sum_{m \in \mathcal{S}}\left(e^{-m r}-\operatorname{Re} e^{-m(r+i y)}\right)=\sum_{m \in \mathcal{S}} e^{-m r}(1-\cos m y) .
$$

Now (M3') yields

and so it suffices to show

$$
G(r, y) \geq c_{2}\left(\log \frac{1}{r}\right)^{2+4 / \alpha} \text { for } \frac{\pi}{2} \leq|y| \leq \pi
$$

$$
G(r, y) \geq c_{5}\left(\log \frac{1}{r}\right)^{2}
$$

for some constant $c_{5}>0$ uniformly for $r^{1+c_{3}} \leq|y| \leq \frac{\pi}{2}$ as $r \rightarrow 0^{+}$.

We split this interval into three parts according to the size of $|y|$.

- $r \leq|y| \leq\left(\log \frac{1}{r}\right)^{-\frac{2}{\alpha}}$ : Note that for $|t| \leq \pi$

$$
1-\cos t \geq \frac{2}{\pi^{2}} t^{2}
$$

Thus we can apply Lemma 8 to find

$$
\begin{aligned}
G(r, y) & \geq \sum_{\substack{1 \leq m \leq|y|^{-1} \\
m \in \mathcal{S}}} e^{-m r}(1-\cos m y) \geq \sum_{\substack{1 \leq m \leq|y|^{-1} \\
m \in \mathcal{S}}} e^{-m r} \frac{2}{\pi^{2}} m^{2} y^{2} \\
& \geq \frac{2}{\pi^{2}} y^{2} \sum_{\substack{1 \leq m \leq|y|^{-1} \\
m \in \mathcal{S}}} e^{-\frac{r}{|y|}} m^{2} \geq \frac{2}{\pi^{2}} e^{-1} y^{2} \cdot c_{6}|y|^{-(\alpha+2)} \\
& \geq c_{5}|y|^{-\alpha} \geq c_{5}\left(\log \frac{1}{r}\right)^{2}
\end{aligned}
$$

if $c_{5}$ is chosen sufficiently small.

- $r^{1+c_{3}} \leq|y| \leq r$ : In the same manner as before, we get

$$
\begin{aligned}
G(r, y) & \geq \sum_{\substack{1 \leq m \leq r^{-1} \\
m \in \mathcal{S}}} e^{-m r}(1-\cos m y) \geq \sum_{\substack{1 \leq m \leq r^{-1} \\
m \in \mathcal{S}}} \frac{2}{\pi^{2}} e^{-1} y^{2} m^{2} \\
& \geq \frac{2}{\pi^{2}} e^{-1} y^{2} \cdot c_{6} r^{-(\alpha+2)} \geq c_{7} r^{2 c_{3}-\alpha} \geq c_{5}\left(\log \frac{1}{r}\right)^{2} .
\end{aligned}
$$

- $\left(\log \frac{1}{r}\right)^{-\frac{2}{\alpha}} \leq|y| \leq \frac{\pi}{2}$ : It is clear that there exists an integer $\ell$ such that

$$
\frac{\pi}{2} \leq 2^{\ell}|y| \leq \pi
$$

From the inequality $1-\cos \theta \geq \frac{1}{4}(1-\cos 2 \theta)$ we get by iteration

$$
1-\cos \theta \geq 4^{-\ell}\left(1-\cos 2^{\ell} \theta\right),
$$


which allows us to apply (M3') again:

$$
\begin{aligned}
G(r, y) & \geq 4^{-\ell} \sum_{m \in \mathcal{S}} e^{-m r}\left(1-\cos 2^{\ell} m y\right) \geq 4^{-\ell} c_{2}\left(\log \frac{1}{r}\right)^{2+4 / \alpha} \\
& \geq \frac{c_{2}}{\pi^{2}} y^{2}\left(\log \frac{1}{r}\right)^{2+4 / \alpha} \geq \frac{c_{2}}{\pi^{2}}\left(\log \frac{1}{r}\right)^{2}
\end{aligned}
$$

so that we obtain the desired estimate in this case as well.

Now we return to the integral representation (3.1): choose $c_{3}$ such that $\frac{\alpha}{3}<c_{3}<\frac{\alpha}{2}$, e.g. $c_{3}=\frac{3 \alpha}{7}$, and split the interval into the part $|t| \leq r^{1+c_{3}}$ and the remaining two intervals. For the latter, Lemma 10 shows that

$$
\int_{r^{1+c_{3}}}^{\pi} \exp (i n t+f(u, r+i t)) \mathrm{d} t \ll \exp \left(f(u, r)-c_{8}\left(\log \frac{1}{r}\right)^{2}\right) \ll \exp \left(f(u, r)-c_{9} \log ^{2} n\right)
$$

for certain positive constants $c_{8}$ and $c_{9}$ (uniformly in $u$ ), and the same estimate holds for $-\pi \leq$ $t \leq-r^{1+c_{3}}$. For the central integral, we have to expand $f(u, r+i t)$ around $t=0$ : by our choice of $r$, the first derivative with respect to $t$ is $-i n$, and the second derivative is given by

$$
-B^{2}=-B^{2}(u, r)=\left.\frac{\partial^{2} f(u, r+i t)}{\partial^{2} t}\right|_{t=0}=-\sum_{m \in \mathcal{S}} \frac{m^{2} u^{-1} e^{r m}}{\left(u^{-1} e^{r m}+1\right)^{2}} .
$$

Now we can apply the Mellin transform technique again: the transform of $\frac{x^{2} u^{-1} e^{x}}{\left(u^{-1} e^{x}+1\right)^{2}}$ is given by

$$
\mathcal{M}\left[\frac{x^{2} u^{-1} e^{x}}{\left(u^{-1} e^{x}+1\right)^{2}} ; s\right]=s(s+1) Y(u, s),
$$

and thus

$$
\mathcal{M}\left[\sum_{m \in \mathcal{S}} \frac{x^{2} m^{2} u^{-1} e^{x m}}{\left(u^{-1} e^{x m}+1\right)^{2}} ; s\right]=s(s+1) Y(u, s) D(s) .
$$

Thus, applying Lemma 6 yields

$$
B^{2}=\sum_{m \in \mathcal{S}} \frac{m^{2} u^{-1} e^{r m}}{\left(u^{-1} e^{r m}+1\right)^{2}}=r^{-(\alpha+2)} \Phi_{2}(u, \omega \log r)+\mathcal{O}\left(r^{-(\alpha+2)+\varepsilon}\right),
$$

where $\Phi_{2}$ is a periodic function; again, a simple argument shows that $\Phi_{2}$ is bounded below by a positive constant (uniformly for $\delta \leq u \leq \delta^{-1}$, as it is the case for all our estimates), implying that $B^{2}$ is of order $r^{-(\alpha+2)}$ : just note that

$$
\begin{aligned}
B^{2} & =\sum_{m \in \mathcal{S}} \frac{m^{2} u^{-1} e^{r m}}{\left(u^{-1} e^{r m}+1\right)^{2}} \geq \sum_{\substack{m \leq r^{-1} \\
m \in \mathcal{S}}} \frac{m^{2} u^{-1} e^{r m}}{\left(u^{-1} e^{r m}+1\right)^{2}} \\
& \geq \sum_{\substack{m \leq r^{-1} \\
m \in \mathcal{S}}} \frac{\delta m^{2}}{\left(\delta^{-1} e+1\right)^{2}} \gg \sum_{\substack{m \leq r^{-1} \\
m \in \mathcal{S}}} m^{2} \gg r^{-(\alpha+2)}
\end{aligned}
$$

by Lemma 8 Finally, we estimate the third derivative as follows: it is given by

$$
\frac{\partial^{3} f(u, r+i t)}{\partial^{3} t}=-i \sum_{m \in \mathcal{S}} \frac{m^{3} u^{-1} e^{m r(1+i t)}\left(1-u^{-1} e^{m r(1+i t)}\right)}{\left(u^{-1} e^{m r(1+i t)}+1\right)^{3}} .
$$


Now let $m_{0}=r^{-\left(1+c_{10}\right)}$ for some constant $c_{10}>0$, and write $v=u^{-1}$ for short. Then we split up the sum into two parts according to whether $m \leq m_{0}$ or not. For the latter we get

$$
\begin{aligned}
& \left|\sum_{\substack{m>m_{0} \\
m \in \mathcal{S}}} \frac{m^{3} v e^{m r(1+i t)}\left(1-v e^{m r(1+i t)}\right)}{\left(v e^{m r(1+i t)}+1\right)^{3}}\right| \leq \sum_{\substack{m>m_{0} \\
m \in \mathcal{S}}} \frac{m^{3} e^{m r}\left(1+v e^{m r}\right)}{\left|v e^{m r(1+i t)}+1\right|^{3}} \\
& \quad \leq \sum_{\substack{m>m_{0} \\
m \in \mathcal{S}}} \frac{m^{3} e^{m r}\left(1+v e^{m r}\right)}{\left(v e^{m r}-1\right)^{3}} \ll \sum_{\substack{m>m_{0} \\
m \in \mathcal{S}}} \frac{m^{3}}{e^{m r}} \ll \frac{r^{-4-3 c_{10}}}{e^{r^{-c_{10}}}} .
\end{aligned}
$$

For the remaining sum we note that

$$
\left|1+v e^{m r(1+i t)}\right| \geq\left(1+v e^{m r}\right) \cos \left(\frac{m r t}{2}\right) .
$$

Therefore we get

$$
\begin{aligned}
& \left|\sum_{\substack{m \leq m_{0} \\
m \in \mathcal{S}}} \frac{m^{3} v e^{m r(1+i t)}\left(1-v e^{m r(1+i t)}\right)}{\left(v e^{m r(1+i t)}+1\right)^{3}}\right| \leq \sum_{\substack{m \leq m_{0} \\
m \in \mathcal{S}}} \frac{m^{3} e^{m r}\left(1+v e^{m r}\right)}{\left|v e^{m r(1+i t)}+1\right|^{3}} \\
& \quad \leq \sum_{\substack{m \leq m_{0} \\
m \in \mathcal{S}}} \frac{m^{3} e^{m r}\left(1+v e^{m r}\right)}{\left(v e^{m r}+1\right)^{3}}\left(1+\mathcal{O}\left((r m t)^{2}\right)\right) \leq \sum_{\substack{m \leq m_{0} \\
m \in \mathcal{S}}} \frac{m^{3}}{v^{2} e^{m r}}\left(1+\mathcal{O}\left((r m t)^{2}\right)\right) \\
& \leq \sum_{m \in \mathcal{S}} \frac{m^{3}}{v^{2} e^{m r}}+\mathcal{O}\left(\sum_{m \in \mathcal{S}} \frac{m^{5} r^{2} t^{2}}{e^{m r}}\right) \ll r^{-3-\alpha}+r^{-3-\alpha} t^{2},
\end{aligned}
$$

where the last estimate is obtained by means of Lemma 6 again. So finally,

$$
\frac{\partial^{3} f(u, r+i t)}{\partial^{3} t} \ll r^{-3-\alpha}
$$

for $|t| \leq r^{1+c_{3}}$, and so we have the expansion

$$
f(u, r+i t)=f(u, r)-i n t-\frac{B^{2}}{2} t^{2}+\mathcal{O}\left(r^{-3-\alpha} t^{3}\right) .
$$

Hence, the corresponding integral can be estimated as follows:

$$
\begin{aligned}
\frac{e^{n r}}{2 \pi} \int_{-r^{1+c_{3}}}^{r^{1+c_{3}}} & \exp (i n t+f(u, r+i t)) \mathrm{d} t \\
& =\frac{e^{n r+f(u, r)}}{2 \pi} \int_{-r^{1+c_{3}}}^{r^{1+c_{3}}} \exp \left(-\frac{B^{2}}{2} t^{2}+\mathcal{O}\left(r^{3 c_{3}-\alpha}\right)\right) \mathrm{d} t \\
& =\frac{e^{n r+f(u, r)}}{2 \pi}\left(\int_{-r^{1+c_{3}}}^{r^{1+c_{3}}} \exp \left(-\frac{B^{2}}{2} t^{2}\right) \mathrm{d} t\right)\left(1+\mathcal{O}\left(r^{2 \alpha / 7}\right)\right) \\
& =\frac{e^{n r+f(u, r)}}{2 \pi}\left(\int_{-r^{1+c_{3}}}^{r^{1+c_{3}}} \exp \left(-\frac{B^{2}}{2} t^{2}\right) \mathrm{d} t\right)\left(1+\mathcal{O}\left(n^{-2 \alpha /(7 \alpha+7)}\right)\right)
\end{aligned}
$$

by our choice of $c_{3}$. Also note that

$$
\begin{aligned}
\int_{-r^{1+c_{3}}}^{r^{1+c_{3}}} \exp \left(-\frac{B^{2}}{2} t^{2}\right) \mathrm{d} t & =\int_{-\infty}^{\infty} \exp \left(-\frac{B^{2}}{2} t^{2}\right) \mathrm{d} t-2 \int_{r^{1+c_{3}}}^{\infty} \exp \left(-\frac{B^{2}}{2} t^{2}\right) \\
& =\frac{\sqrt{2 \pi}}{B}+\mathcal{O}\left(\int_{r^{1+c_{3}}}^{\infty} \exp \left(-\frac{B^{2} r^{1+c_{3}}}{2} t\right) \mathrm{d} t\right) \\
& =\frac{\sqrt{2 \pi}}{B}+\mathcal{O}\left(r^{-1-c_{3}} B^{-2} \exp \left(-\frac{B^{2} r^{2\left(1+c_{3}\right)}}{2}\right)\right) .
\end{aligned}
$$


We know that $B^{2} \gg r^{-(\alpha+2)}$, which implies

$$
B^{2} r^{2\left(1+c_{3}\right)} \gg r^{2 c_{3}-\alpha}=r^{-\alpha / 7} \gg n^{-\alpha /(7 \alpha+7)} .
$$

Hence, the error term tends to 0 faster than any power of $n$.

Putting everything together, we find that

$$
Q_{n}(u)=\frac{1}{\sqrt{2 \pi B^{2}}} e^{n r+f(u, r)}\left(1+\mathcal{O}\left(n^{-2 \alpha /(7 \alpha+7)}\right)\right)
$$

uniformly in $u$. Now we study the moment generating function of the random variable $\varpi_{n}$ (the number of parts in a random partition), which can be expressed in terms of $Q_{n}(u)$ : let $M_{n}(t)=$ $\mathbb{E}\left(e^{\left(\varpi_{n}-\mu_{n}\right) t / \sigma_{n}}\right)$, where $t$ is real and $\mu_{n}$ and $\sigma_{n}$ are chosen as in (2.3) and (2.4). Then we get

$$
\begin{aligned}
M_{n}(t)= & \exp \left(-\frac{\mu_{n} t}{\sigma_{n}}\right) \frac{Q_{n}\left(e^{t / \sigma_{n}}\right)}{Q_{n}(1)} \\
= & \sqrt{\frac{B^{2}(1, r(n, 1))}{B^{2}\left(e^{t / \sigma_{n}}, r\left(n, e^{t / \sigma_{n}}\right)\right)}} \exp \left(-\frac{\mu_{n} t}{\sigma_{n}}+n r\left(n, e^{t / \sigma_{n}}\right)+f\left(e^{t / \sigma_{n}}, r\left(n, e^{t / \sigma_{n}}\right)\right)\right. \\
& \left.-n r(n, 1)-f(1, r(n, 1))+\mathcal{O}\left(n^{-2 \alpha /(7 \alpha+7)}\right)\right) .
\end{aligned}
$$

Now, we want to determine the expansion around $t=0$; for this purpose, we need the partial derivatives of $r(n, u)$ with respect to $u$ : recall that $r=r(n, u)$ was defined by

$$
n=-f_{\tau}(u, r)=\sum_{m \in \mathcal{S}} \frac{m}{u^{-1} e^{r m}+1} .
$$

Hence, the partial derivatives can be determined by means of implicit differentiation:

$$
r_{u}=r_{u}(n, u)=-\frac{f_{u \tau}(u, r)}{f_{\tau \tau}(u, r)}=\frac{\sum_{m \in \mathcal{S}} \frac{m e^{m r}}{\left(e^{m r}+u\right)^{2}}}{\sum_{m \in \mathcal{S}} \frac{u m^{2} e^{m r}}{\left(e^{m r}+u\right)^{2}}}
$$

and similarly

$$
\begin{aligned}
r_{u u}=r_{u u}(n, u)= & \frac{-}{f_{\tau \tau \tau}(u, r) f_{u \tau}(u, r)^{2}+2 f_{u \tau \tau}(u, r) f_{u \tau}(u, r) f_{\tau \tau}(u, r)-f_{u u \tau}(u, r) f_{\tau \tau}(u, r)^{2}} \\
r_{\tau \tau}(u, r)^{3} & r_{u u u}(n, u)=f_{\tau \tau}(u, r)^{-5}\left(-f_{u u u \tau}(u, r) f_{\tau \tau}(u, r)^{4}\right. \\
& +\left(3 f_{u u \tau \tau}(u, r) f_{u \tau}(u, r)+3 f_{u u \tau}(u, r) f_{u \tau \tau}(u, r)\right) f_{\tau \tau}(u, r)^{3} \\
& +\left(-3 f_{u \tau \tau \tau}(u, r) f_{u \tau}(u, r)^{2}-6 f_{u \tau \tau}(u, r)^{2} f_{u \tau}(u, r)\right. \\
& \left.-3 f_{u u \tau}(u, r) f_{\tau \tau \tau}(u, r) f_{u \tau}(u, r)\right) f_{\tau \tau}(u, r)^{2} \\
& +\left(f_{\tau \tau \tau \tau}(u, r) f_{u \tau}(u, r)^{3}+9 f_{u \tau \tau}(u, r) f_{\tau \tau \tau}(u, r) f_{u \tau}(u, r)^{2}\right) f_{\tau \tau}(u, r) \\
& \left.-3 f_{u \tau}(u, r)^{3} f_{\tau \tau \tau}(u, r)^{2}\right),
\end{aligned}
$$

where the derivatives of $f$ are given as follows:

$$
\begin{aligned}
f_{u \tau}(u, r) & =-\sum_{m \in \mathcal{S}} \frac{m e^{m r}}{\left(e^{m r}+u\right)^{2}} \ll r^{-(1+\alpha)} \ll n, \\
f_{\tau \tau}(u, r) & =\sum_{m \in \mathcal{S}} \frac{u m^{2} e^{m r}}{\left(e^{m r}+u\right)^{2}} \ll r^{-(2+\alpha)} \ll n^{1+1 /(1+\alpha)}, \\
f_{u u \tau}(u, r) & =\sum_{m \in \mathcal{S}} \frac{2 m e^{m r}}{\left(e^{m r}+u\right)^{3}} \ll r^{-(1+\alpha)} \ll n, \\
f_{u \tau \tau}(u, r) & =\sum_{m \in \mathcal{S}} \frac{m^{2} e^{m r}\left(e^{m r}-u\right)}{\left(e^{m r}+u\right)^{3}} \ll r^{-(2+\alpha)} \ll n^{1+1 /(1+\alpha)}, \\
f_{\tau \tau \tau}(u, r) & =-\sum_{m \in \mathcal{S}} \frac{u m^{3} e^{m r}\left(e^{m r}-u\right)}{\left(e^{m r}+u\right)^{3}} \ll r^{-(3+\alpha)} \ll n^{1+2 /(1+\alpha)},
\end{aligned}
$$




$$
\begin{aligned}
& f_{u u u \tau}(u, r)=-\sum_{m \in \mathcal{S}} \frac{6 m e^{m r}}{\left(e^{m r}+u\right)^{4}} \ll r^{-(1+\alpha)} \ll n \\
& f_{u u \tau \tau}(u, r)=-\sum_{m \in \mathcal{S}} \frac{2 m^{2} e^{m r}\left(2 e^{m r}-u\right)}{\left(e^{m r}+u\right)^{4}} \ll r^{-(2+\alpha)} \ll n^{1+1 /(1+\alpha)}, \\
& f_{u \tau \tau \tau}(u, r)=-\sum_{m \in \mathcal{S}} \frac{m^{3} e^{m r}\left(e^{2 m r}-4 u e^{m r}+u^{2}\right)}{\left(e^{m r}+u\right)^{4}} \ll r^{-(3+\alpha)} \ll n^{1+2 /(1+\alpha)}, \\
& f_{\tau \tau \tau \tau}(u, r)=\sum_{m \in \mathcal{S}} \frac{u m^{4} e^{m r}\left(e^{2 m r}-4 u e^{m r}+u^{2}\right)}{\left(e^{m r}+u\right)^{4}} \ll r^{-(4+\alpha)} \ll n^{1+3 /(1+\alpha)} .
\end{aligned}
$$

The asymptotic estimates are all obtained by means of the usual Mellin transform method. Furthermore, note that

$$
f_{\tau \tau}(u, r)=B^{2}(u, r) \gg r^{-(2+\alpha)} \gg n^{1+1 /(1+\alpha)},
$$

from which it follows that $r_{u}, r_{u u}, r_{u u u} \ll n^{-1 /(1+\alpha)}$, all uniformly in $u$. Thus, we have the following expansions:

$$
r\left(n, e^{t / \sigma_{n}}\right)-r(n, 1)=r_{u}(n, 1) \cdot \frac{t}{\sigma_{n}}+\frac{r_{u}(n, 1)+r_{u u}(n, 1)}{2} \cdot\left(\frac{t}{\sigma_{n}}\right)^{2}+\mathcal{O}\left(n^{-1 /(1+\alpha)} \frac{t^{3}}{\sigma_{n}^{3}}\right)
$$

and

$$
\begin{aligned}
f\left(e^{t / \sigma_{n}}, r\left(n, e^{t / \sigma_{n}}\right)\right)-f(1, r(n, 1))=\left(f_{\tau}(1, r(n, 1)) r_{u}(n, 1)+f_{u}(1, r(n, 1))\right) \cdot \frac{t}{\sigma_{n}} \\
+\frac{1}{2}\left(f_{\tau}(1, r(n, 1))\left(r_{u}(n, 1)+r_{u u}(n, 1)\right)+f_{\tau \tau}(1, r(n, 1)) r_{u}(n, 1)^{2}+2 f_{u \tau}(1, r(n, 1)) r_{u}(n, 1)\right. \\
\left.+f_{u}(1, r(n, 1))+f_{u u}(1, r(n, 1))\right) \cdot\left(\frac{t}{\sigma_{n}}\right)^{2}+\mathcal{O}\left(n^{\alpha /(1+\alpha)} \frac{t^{3}}{\sigma_{n}^{3}}\right) .
\end{aligned}
$$

Altogether, this means that the exponent in (3.3) can be written as

$$
\begin{aligned}
& \left(n r_{u}(n, 1)+f_{\tau}(1, \eta) r_{u}(n, 1)+f_{u}(1, \eta)-\mu_{n}\right) \cdot \frac{t}{\sigma_{n}} \\
& +\frac{1}{2}\left(n\left(r_{u}(n, 1)+r_{u u}(n, 1)\right)+f_{\tau}(1, \eta)\left(r_{u}(n, 1)+r_{u u}(n, 1)\right)+f_{\tau \tau}(1, \eta) r_{u}(n, 1)^{2}\right. \\
& \left.\quad+2 f_{u \tau}(1, \eta) r_{u}(n, 1)+f_{u}(1, \eta)+f_{u u}(1, \eta)\right) \cdot\left(\frac{t}{\sigma_{n}}\right)^{2}+\mathcal{O}\left(n^{\alpha /(1+\alpha)} \frac{t^{3}}{\sigma_{n}^{3}}+n^{-2 \alpha /(7 \alpha+7)}\right),
\end{aligned}
$$

where we use $\eta$ as an abbreviation for $r(n, 1)$. Now, we make use of the fact that $n=-f_{\tau}(1, \eta)$ and that $r_{u}(n, 1)=-\frac{f_{u \tau}(1, \eta)}{f_{\tau \tau}(1, \eta)}$ to simplify this expression:

$$
\begin{aligned}
\left(f_{u}(1, \eta)-\mu_{n}\right) \cdot \frac{t}{\sigma_{n}}+\frac{1}{2}\left(f_{u}(1, \eta)+f_{u u}(1, \eta)-\frac{f_{u \tau}(1, \eta)^{2}}{f_{\tau \tau}(1, \eta)}\right) & \left(\frac{t}{\sigma_{n}}\right)^{2} \\
& +\mathcal{O}\left(n^{\alpha /(1+\alpha)} \frac{t^{3}}{\sigma_{n}^{3}}+n^{-2 \alpha /(7 \alpha+7)}\right)
\end{aligned}
$$

It is not difficult to show in a similar way that

$$
\frac{B^{2}(1, r(n, 1))}{B^{2}\left(e^{t / \sigma_{n}}, r\left(n, e^{t / \sigma_{n}}\right)\right)}=1+\mathcal{O}\left(\frac{t}{\sigma_{n}}\right)
$$

and so we obtain the following asymptotic formula for the moment generating function from (3.3):

$$
\begin{aligned}
M_{n}(t)=\exp \left(\left(f_{u}(1, \eta)-\mu_{n}\right) \cdot \frac{t}{\sigma_{n}}+\frac{1}{2}\left(f_{u}(1, \eta)\right.\right. & \left.+f_{u u}(1, \eta)-\frac{f_{u \tau}(1, \eta)^{2}}{f_{\tau \tau}(1, \eta)}\right) \cdot\left(\frac{t}{\sigma_{n}}\right)^{2} \\
& \left.+\mathcal{O}\left(\frac{t}{\sigma_{n}}+n^{\alpha /(1+\alpha)} \frac{t^{3}}{\sigma_{n}^{3}}+n^{-2 \alpha /(7 \alpha+7)}\right)\right)
\end{aligned}
$$


Now, note that $\mu_{n}$ and $\sigma_{n}$ were chosen in such a way that

$$
\begin{aligned}
& \mu_{n}=f_{u}(1, \eta)=\sum_{m \in \mathcal{S}} \frac{1}{e^{\eta m}+1}, \\
& \sigma_{n}^{2}=f_{u}(1, \eta)+f_{u u}(1, \eta)-\frac{f_{u \tau}(1, \eta)^{2}}{f_{\tau \tau}(1, \eta)}=\sum_{m \in \mathcal{S}} \frac{e^{\eta m}}{\left(e^{\eta m}+1\right)^{2}}-\frac{\left(\sum_{m \in \mathcal{S}} \frac{m e^{\eta m}}{\left(e^{\eta m}+1\right)^{2}}\right)^{2}}{\sum_{m \in \mathcal{S}} \frac{m^{2} e^{\eta m}}{\left(e^{\eta m}+1\right)^{2}}} .
\end{aligned}
$$

We only have to prove that the error term is small, and so we need a lower estimate for $\sigma_{n}$ : first of all, our usual Mellin transform technique shows that

$$
\mu_{n}=\eta^{-\alpha} \Phi_{\mu}(\omega \log \eta)+\mathcal{O}\left(\eta^{-\alpha+\varepsilon}\right) \sim n^{\alpha /(1+\alpha)} \Psi_{\mu}\left(\frac{\omega \log n}{\alpha+1}\right)
$$

and

$$
\sigma_{n}^{2}=\eta^{-\alpha} \Phi_{\sigma}(\omega \log \eta)+\mathcal{O}\left(\eta^{-\alpha+\varepsilon}\right) \sim n^{\alpha /(1+\alpha)} \Psi_{\sigma}\left(\frac{\omega \log n}{\alpha+1}\right)
$$

for certain 1-periodic functions $\Phi_{\mu}, \Phi_{\sigma}$ and $\Psi_{\mu}, \Psi_{\sigma}$ (note that since $\log \eta \sim-\frac{\log n}{\alpha+1}$, the periods differ by a factor of $\alpha+1$ ).

$\mu_{n}=\sum_{m \in \mathcal{S}} \frac{1}{e^{\eta m}+1}$ is obviously a positive monotonic function of $\eta$, showing immediately that $\Phi_{\mu}$ and $\Psi_{\mu}$ must be bounded above and below by positive constants (in the same way as it was shown that $\left.r \gg n^{-1 /(1+\alpha)}\right)$. However, this approach does not apply so easily to $\sigma_{n}$ (in particular, it is less obvious that $\Phi_{\sigma}$ cannot be identically 0 ), so we proceed a little differently: using the abbreviation $q(x)=\frac{e^{x}}{\left(e^{x}+1\right)^{2}}$, we can write the numerator as

$$
\begin{aligned}
\left(\sum_{m \in \mathcal{S}} \frac{e^{\eta m}}{\left(e^{\eta m}+1\right)^{2}}\right) & \left(\sum_{m \in \mathcal{S}} \frac{m^{2} e^{\eta m}}{\left(e^{\eta m}+1\right)^{2}}\right)-\left(\sum_{m \in \mathcal{S}} \frac{m e^{\eta m}}{\left(e^{\eta m}+1\right)^{2}}\right)^{2} \\
& =\sum_{m_{1} \in \mathcal{S}} \sum_{m_{2} \in \mathcal{S}}\left(m_{2}^{2}-m_{1} m_{2}\right) q\left(\eta m_{1}\right) q\left(\eta m_{2}\right) \\
& =\sum_{m_{1} \in \mathcal{S}} \sum_{\substack{m_{2}>m_{1} \\
m_{2} \in \mathcal{S}}}\left(m_{2}-m_{1}\right)^{2} q\left(\eta m_{1}\right) q\left(\eta m_{2}\right) \\
& =\frac{1}{2} \sum_{m_{1} \in \mathcal{S}} \sum_{m_{2} \in \mathcal{S}}\left(m_{2}-m_{1}\right)^{2} q\left(\eta m_{1}\right) q\left(\eta m_{2}\right) .
\end{aligned}
$$

This can be estimated as follows:

$$
\begin{aligned}
& \frac{1}{2} \sum_{m_{1} \in \mathcal{S}} \sum_{m_{2} \in \mathcal{S}}\left(m_{2}-m_{1}\right)^{2} q\left(\eta m_{1}\right) q\left(\eta m_{2}\right) \geq \frac{1}{2} \sum_{\substack{m_{1} \leq \eta^{-1} / 2 \\
m_{1} \in \mathcal{S}}} \sum_{\substack{\eta^{-1} \leq m_{2} \leq C \eta^{-1} \\
m_{2} \in \mathcal{S}}}\left(m_{2}-m_{1}\right)^{2} q\left(\eta m_{1}\right) q\left(\eta m_{2}\right) \\
& \geq \frac{1}{2} \sum_{\substack{m_{1} \leq \eta^{-1} / 2 \\
m_{1} \in \mathcal{S}}} \sum_{\substack{\eta^{-1} \leq m_{2} \leq C \eta^{-1} \\
m_{2} \in \mathcal{S}}} \frac{1}{4 \eta^{2}} q\left(\eta m_{1}\right) q\left(\eta m_{2}\right) \\
& \gg \eta^{-2}\left(\sum_{\substack{m_{1} \leq \eta^{-1} / 2 \\
m_{1} \in \mathcal{S}}} 1\right)\left(\sum_{\substack{\eta^{-1} \leq m_{2} \leq C \eta^{-1} \\
m_{2} \in \mathcal{S}}} 1\right) \\
& \gg \eta^{-2-2 \alpha}
\end{aligned}
$$


by Lemma 8 and the corollary thereafter. The denominator has already been shown earlier to be of order $\eta^{-2-\alpha}$. Hence, $\sigma_{n}^{2} \gg \eta^{-\alpha} \gg n^{\alpha /(1+\alpha)}$. Putting everything together, we arrive at

$$
\begin{aligned}
M_{n}(t) & =\exp \left(\frac{t^{2}}{2}+\mathcal{O}\left(n^{-\alpha /(2+2 \alpha)}\left(t+t^{3}\right)+n^{-2 \alpha /(7 \alpha+7)}\right)\right) \\
& =\exp \left(\frac{t^{2}}{2}+\mathcal{O}\left(n^{-2 \alpha /(7 \alpha+7)}\right)\right)
\end{aligned}
$$

for bounded $t$. Now, Curtiss's Theorem [3] shows that the distribution of $\varpi_{n}$ is indeed asymptotically normal. For the remaining parts of the theorem, we can again follow the lines of Hwang [12]: note that if $t=o\left(n^{\alpha /(6 \alpha+6)}\right)$, the above equation, together with Markov's inequality, yields

$$
\begin{aligned}
\mathbb{P}\left(\frac{\varpi_{n}-\mu_{n}}{\sigma_{n}} \geq x\right) & \leq e^{-t x} M_{n}(t) \\
& =e^{-t x+t^{2} / 2}\left(1+\mathcal{O}\left(n^{-\alpha /(2+2 \alpha)}\left(t+t^{3}\right)+n^{-2 \alpha /(7 \alpha+7)}\right)\right) .
\end{aligned}
$$

We set $T=n^{\alpha /(6 \alpha+6)} / \log n$ and $t=x$ for $x \leq T$ (minimizing $\left.-t x+t^{2} / 2\right)$ to obtain

$$
\mathbb{P}\left(\frac{\varpi_{n}-\mu_{n}}{\sigma_{n}} \geq x\right) \leq e^{-x^{2} / 2}\left(1+\mathcal{O}\left((\log n)^{-3}\right)\right)
$$

and for $x \geq T$, by setting $t=T$,

$$
\mathbb{P}\left(\frac{\varpi_{n}-\mu_{n}}{\sigma_{n}} \geq x\right) \leq e^{-T x / 2}\left(1+\mathcal{O}\left((\log n)^{-3}\right)\right) .
$$

The probability $\mathbb{P}\left(\frac{\varpi_{n}-\mu_{n}}{\sigma_{n}} \leq-x\right)$ can be estimated in an analogous way. Finally, we can also apply Hwang's method that was used in [12] to show that the mean and variance of $\varpi_{n}$ are indeed asymptotic to $\mu_{n}$ and $\sigma_{n}^{2}$ respectively.

Remark 1. If the only pole of the Dirichlet series $D(s)$ is at $s=\alpha$ (so that the periodic functions $\Phi_{\mu}$ and $\Phi_{\sigma}$ are actually constant), we obtain the asymptotic expressions for $\mu_{n}$ and $\sigma_{n}^{2}$ given in Theorem [2, in this case, Lemma 6 yields

$$
n=\sum_{m \in \mathcal{S}} \frac{m}{e^{\eta m}+1} \sim A_{0}\left(1-2^{-\alpha}\right) \Gamma(\alpha+1) \zeta(\alpha+1) \eta^{-(\alpha+1)}=\kappa \alpha \eta^{-(\alpha+1)},
$$

where $\kappa$ is taken as in Theorem 2 , and

$$
\begin{aligned}
\mu_{n}=\sum_{m \in \mathcal{S}} \frac{1}{e^{\eta m}+1} & \sim A_{0}\left(1-2^{1-\alpha}\right) \Gamma(\alpha) \zeta(\alpha) \eta^{-\alpha} \\
& \sim A_{0}\left(1-2^{1-\alpha}\right) \Gamma(\alpha) \zeta(\alpha)(\kappa \alpha)^{-\alpha /(\alpha+1)} n^{\alpha /(\alpha+1)} \\
& =(\kappa \alpha)^{1 /(\alpha+1)} \frac{\left(1-2^{1-\alpha}\right) \zeta(\alpha)}{\alpha\left(1-2^{-\alpha}\right) \zeta(\alpha+1)} n^{\alpha /(\alpha+1)}
\end{aligned}
$$

and an asymptotic formula for $\sigma_{n}^{2}$ follows in a similar manner.

Remark 2. It might be possible to relax the conditions of our theorem, in particular (M1'), even further, so that the poles don't necessarily have to be evenly spaced. However, we are not aware of any natural example for which this actually occurs. Finally, the core of the proof, which is the application of the saddle point method, is in principle also applicable if the Dirichlet series has more complicated singularities (e.g. if $\mathcal{S}$ is the set of all primes), as long as one is able to obtain sufficiently strong upper and lower estimates for the harmonic sums involved.

\section{EXAMPLES}

As mentioned in the introduction, our initial motivating example was the set of integers with certain missing digits. However, there are also other natural examples for which Theorem 5 is applicable. At the end of this section, we also exhibit an example where our theorem fails because one of the conditions doesn't hold. 


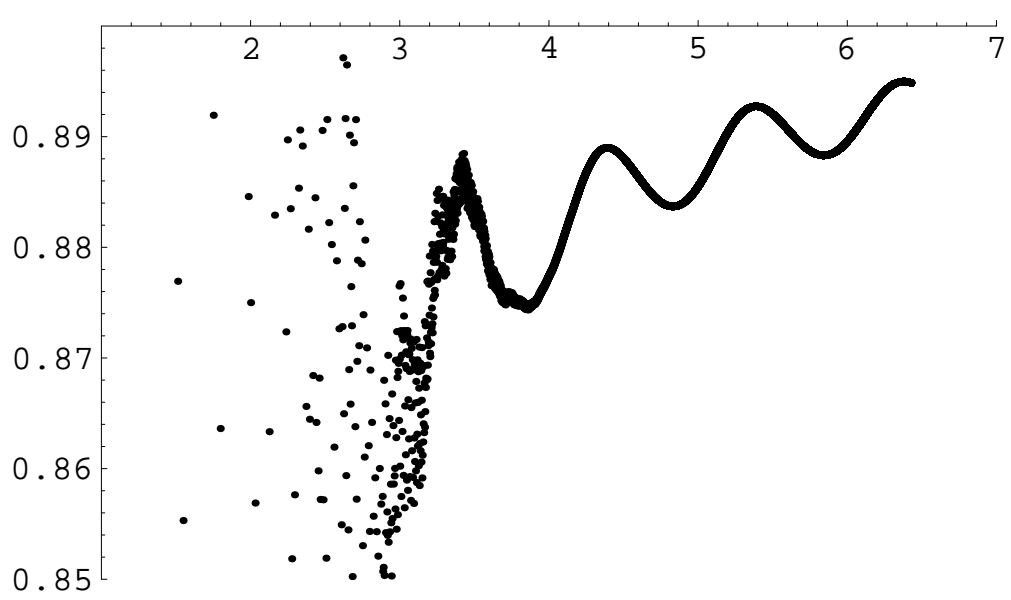

Figure 1. Periodic fluctuations of the mean in an example

4.1. Missing digits. Recall that $\mathcal{M D}(b, D)$ denotes the set of positive integers with the property that all digits in the $b$-ary representation come from the set $D$. Lemma 3 shows immediately that condition (M1') is satisfied, since we have simple poles at $\alpha+2 \pi i k \omega$ with $\alpha=\frac{\log |D|}{\log b}$ and $\omega=(\log b)^{-1}$. (M2') is also obvious in view of Lemma 3 . Condition (M3') can also be proved by elementary means: for $r>0$ and $\frac{\pi}{2} \leq|y| \leq \pi$, choose $k$ in such a way that $b^{k} \leq r^{-1}<b^{k+1}$. Then we have

$$
\begin{aligned}
g(r) & -\operatorname{Re} g(r+i y)=\sum_{m \in \mathcal{S}} e^{-m r}(1-\cos (m y)) \geq \sum_{\substack{m<b^{k} \\
m \in \mathcal{S}}} e^{-m r}(1-\cos (m y)) \\
& \geq e^{-1} \sum_{\substack{m<b^{k} \\
m \in \mathcal{S}}}(1-\cos (m y))=e^{-1} \operatorname{Re} \sum_{\substack{m<b^{k} \\
m \in \mathcal{S}}}(1-\exp (i m y)) \\
& =e^{-1} \operatorname{Re}\left(|D|^{k}-\prod_{j=0}^{k-1} \sum_{d \in D} \exp \left(i d b^{j} y\right)\right) \geq e^{-1}\left(|D|^{k}-\left|\prod_{j=0}^{k-1} \sum_{d \in D} \exp \left(i d b^{j} y\right)\right|\right) \\
& \geq e^{-1}\left(|D|^{k}-\left|\sum_{d \in D} \exp (i d y)\right||D|^{k-1}\right) \geq e^{-1}\left(1-\frac{1}{|D|} \sup _{\frac{\pi}{2} \leq|y| \leq \pi}\left|\sum_{d \in D} \exp (i d y)\right|\right)|D|^{k} \\
& \gg r^{-\log |D| / \log b},
\end{aligned}
$$

which proves (M3'). Note that

$$
\left|\sum_{d \in D} \exp (i d y)\right|<|D|
$$

for $\frac{\pi}{2} \leq|y| \leq \pi$ : Since it was assumed that the digits in $D$ don't have a common divisor $>1, d y$ cannot be a multiple of $2 \pi$ for all $d$.

Figure1 illustrates the periodic fluctuations in an example: here, the set of integers which don't contain the digit 2 in their ternary representation is considered. The plot shows the normalized mean of the length (i.e. $n^{-\alpha /(1+\alpha)} \mathbb{E}\left(\varpi_{n}\right)$, where $\alpha=\frac{\log 2}{\log 3}$ in this case) on a logarithmic scale.

4.2. Missing blocks. The preceding example can easily be extended to integers with missing blocks in their digital expansion, such as the so-called "Fibbinary numbers" (see [18, sequence A003714, or [1]), i.e., integers whose binary representation doesn't contain the block 11: 
$\{1,2,4,5,8,9,10,16, \ldots\}$. Write $\mathcal{F}$ for this set:

$$
\mathcal{F}:=\left\{\sum_{i=0}^{k} a_{i} 2^{i} \mid k \in \mathbb{N}, a_{i} \in\{0,1\}, a_{i} \cdot a_{i+1}=0\right\} .
$$

It is not difficult to show that the associated Dirichlet series satisfies our hypotheses (M1')-(M3'): noting that

$$
\mathcal{F}=2 \mathcal{F} \cup(4 \mathcal{F}+1) \cup\{1\},
$$

we get

$$
\begin{aligned}
\left(1-2^{-s}-4^{-s}\right) D(s) & =1+\sum_{m \in \mathcal{F}}(4 m+1)^{-s}-(4 m)^{-s} \\
& \ll 1+|s| \sum_{m \in \mathcal{F}} m^{-(\operatorname{Re} s+1)},
\end{aligned}
$$

which converges for $\operatorname{Re} s>0$. Therefore, we have

$$
D(s)=\left(1-2^{-s}-4^{-s}\right) R(s)
$$

for a Dirichlet series $R(s)$ that satisfies $R(s) \ll|s|$ uniformly for Re $s \geq \varepsilon$. Hence $\alpha=\frac{\log ((\sqrt{5}+1) / 2)}{\log 2}$, and $\omega=\frac{1}{\log 2}$. Conditions (M1') and (M2') are satisfied as before. Furthermore, in order to prove that (M3') is also fulfilled, we note that

$$
\mathcal{M D}(4,\{0,1\}) \subseteq \mathcal{F}
$$

and so the considerations of the previous example show that

$$
g(r)-\operatorname{Re} g(r+i y)=\sum_{m \in \mathcal{F}} e^{-m r}(1-\cos m y) \geq \sum_{m \in \mathcal{M} \mathcal{D}(4,\{0,1\})} e^{-m r}(1-\cos m y) \gg r^{-1 / 2} .
$$

4.3. Numbers with even/odd length. Let us now consider numbers whose $b$-ary representation has odd length: we obtain the sequence

$$
\{1,4,5,6,7,16,17, \ldots\}
$$

in the binary case, which is Sloane's A053738 [18]. Of course, this example can be generalized in many directions as well. Write $\mathcal{L}$ for the set of all such numbers, i.e.

$$
\mathcal{L}:=\left\{\sum_{i=0}^{2 k} a_{i} b^{i} \mid k \in \mathbb{N}, a_{i} \in\{0,1, \ldots, b-1\}, a_{2 k} \neq 0\right\} .
$$

Thus we get for the Dirichlet generating function

$$
D(s)=\sum_{k \geq 0} \sum_{m=b^{2 k}}^{b^{2 k+1}-1} m^{-s} .
$$

Noting that

$$
\mathcal{L}=\bigcup_{i=0}^{b^{2}-1}\left(b^{2} \mathcal{L}+i\right) \cup\{1, \ldots, b-1\}
$$

we find

$$
\begin{aligned}
D(s) & =\sum_{i=0}^{b^{2}-1} \sum_{m \in \mathcal{L}}\left(b^{2} m+i\right)^{-s}+\sum_{m=1}^{b-1} m^{-s} \\
& =b^{2-2 s} D(s)+\sum_{m=1}^{b-1} m^{-s}+\sum_{i=0}^{b^{2}-1} \sum_{m \in \mathcal{L}}\left(\left(b^{2} m+i\right)^{-s}-\left(b^{2} m\right)^{-s}\right) \\
& =b^{2-2 s} D(s)+R(s) .
\end{aligned}
$$


By the same method as above we see that $R(s)$ converges for $\operatorname{Re} s>0$, and we get

$$
D(s)=\left(1-b^{2-2 s}\right)^{-1} R(s)
$$

which has poles at $s=1+\frac{\pi i}{\log b}$. As before, (M1') and (M2') hold with $\alpha=1$ and $\omega=\frac{1}{2 \log b}$. In order to prove that (M3') holds as well, we can use an elementary argument: choose $K$ such that $b^{2 K+1} \leq r^{-1}<b^{2 K+3}$. Then we have

$$
\begin{aligned}
g(r)-\operatorname{Re} g(r+i y) & =\sum_{k \geq 0} \sum_{m=b^{2 k}}^{b^{2 k+1}-1} e^{-m r}(1-\cos m y) \geq \sum_{m=b^{2 K}}^{b^{2 K+1}-1} e^{-m r}(1-\cos m y) \\
& \geq e^{-1} \sum_{m=b^{2 K}}^{b^{2 K+1}-1}(1-\cos m y) \\
& =e^{-1}\left((b-1) b^{2 K}-\frac{\sin \left(\left(b^{2 K+1}-1 / 2\right) y\right)-\sin \left(\left(b^{2 K}-1 / 2\right) y\right)}{2 \sin (y / 2)}\right) \\
& =e^{-1}(b-1) b^{2 K}+\mathcal{O}(1) \gg r^{-1} .
\end{aligned}
$$

4.4. Numbers with restricted sum of digits. Numbers whose $b$-ary sum of digits has to satisfy a certain congruence have been studied by Gel'fond [10] and Mauduit and Sárközy [14]. Their additive properties have been discussed in a paper by Thuswaldner and Tichy 20 and subsequent papers. This is actually an example for which there is only a single pole: it is not difficult to show that the Dirichlet series associated with the set of all integers whose $b$-ary sum of digits is $\equiv h \bmod k$ is essentially $\frac{1}{k} \zeta(s)$. Hence, $\alpha=1$, and the periodic functions $\Psi_{\mu}$ and $\Psi_{\sigma}$ are actually constants. Hwang's Theorem 2 is still not applicable, however, since there is an additional pole at 0 . Let us illustrate this in the binary case: let $\mathcal{C}_{0}$ and $\mathcal{C}_{1}$ be the sets of integers for which the binary sum of digits is even resp. odd, and let $D_{0}(s)$ and $D_{1}(s)$ be the associated Dirichlet series. Then,

$$
\mathcal{C}_{0}=2 \mathcal{C}_{0} \cup\left(2 \mathcal{C}_{1}+1\right)
$$

and thus

$$
\begin{aligned}
D_{0}(s) & =\sum_{m \in \mathcal{C}_{0}}(2 m)^{-s}+\sum_{m \in \mathcal{C}_{1}}(2 m+1)^{-s}=2^{-s}\left(D_{0}(s)+D_{1}(s)\right)+\sum_{m \in \mathcal{C}_{1}}\left((2 m+1)^{-s}-(2 m)^{-s}\right) \\
& =2^{-s} \zeta(s)+\sum_{m \in \mathcal{C}_{1}}(2 m)^{-s} \sum_{k \geq 1}\left(\begin{array}{c}
-s \\
k
\end{array}\right)(2 m)^{-k}=2^{-s} \zeta(s)+\sum_{k \geq 1}\left(\begin{array}{c}
-s \\
k
\end{array}\right) 2^{-(s+k)} D_{1}(s+k),
\end{aligned}
$$

which obviously has a pole at 0 .

Remark 3. In all the aforementioned examples, one can also work with the squares, cubes, etc. of the numbers in $\mathcal{S}$, since the associated Dirichlet series is essentially the same. There are many other examples of fairly natural sets of integers whose associated Dirichlet series has equidistant poles on a line of the form $\operatorname{Re} s=\alpha$ : for instance, consider palindromes: the binary palindromes are

$$
1,3,5,7,9,15,17,21, \ldots
$$

(Sloane's A006995 18). Since all of them are odd, the length of a partition of $n$ will always have the same parity as $n$, but the central limit theorem still holds. Another example are numbers whose digital representation is the juxtaposition of two identical strings: in base 2, these are

$$
3,10,15,36,45,54,63,136, \ldots
$$

(Sloane's A020330 [18]). In all these cases, (M1') and (M2') are fairly easy to check, proving (M3') is the difficult part.

Let us finally consider a trivial example for which (M1') and (M2') are satisfied, but (M3') isn't: 
4.5. A counterexample. Let us consider the sequence $1,4,4,16,16,16,16,64, \ldots$, i.e. $4^{k}$ appears with multiplicity $2^{k}$. The corresponding Dirichlet series is extremely simple and obviously satisfies (M1') and (M2') (with $\alpha=\frac{1}{2}$ and $\omega=\frac{1}{2 \log 2}$ ):

$$
D(s)=\sum_{k \geq 0} \frac{2^{k}}{4^{k s}}=\frac{1}{1-2^{1-2 s}}
$$

(M3') is violated, however, and there are even infinitely many integers which cannot be partitioned in this case (all those which are $\equiv 2,3 \bmod 4$, for instance), so that Theorem 5 cannot hold any longer.

\section{ACKNOWLEDGMENT}

We would like to thank Peter Grabner for his kind help with the proof of Lemma 8

This paper was written while M. Madritsch was a visitor at the Centre of Experimental Mathematics at the University of Stellenbosch. He thanks the centre for its hospitality. He is also supported by the Austrian Science Foundation FWF, project S9610, that is part of the Austrian National Research Network "Analytic Combinatorics and Probabilistic Number Theory".

\section{REFERENCES}

[1] J.-P. Allouche, J. Shallit, and G. Skordev. Self-generating sets, integers with missing blocks, and substitutions. Discrete Math., 292(1-3):1-15, 2005.

[2] G. E. Andrews. The theory of partitions. Addison-Wesley Publishing Co., Reading, Mass.-London-Amsterdam, 1976. Encyclopedia of Mathematics and its Applications, Vol. 2.

[3] J. Curtiss. A note on the theory of moment generating functions. The Annals of Mathematical Statistics, 13:430-433, 1942.

[4] P. Erdös and J. Lehner. The distribution of the number of summands in the partitions of a positive integer. Duke Math. J., 8:335-345, 1941.

[5] P. Erdős, C. Mauduit, and A. Sárközy. On arithmetic properties of integers with missing digits. I. Distribution in residue classes. J. Number Theory, 70(2):99-120, 1998.

[6] P. Erdős, C. Mauduit, and A. Sárközy. On arithmetic properties of integers with missing digits. II. Prime factors. Discrete Math., 200(1-3):149-164, 1999. Paul Erdős memorial collection.

[7] P. Erdős and M. Szalay. On the statistical theory of partitions. In Topics in classical number theory, Vol. I, II (Budapest, 1981), volume 34 of Colloq. Math. Soc. János Bolyai, pages 397-450. North-Holland, Amsterdam, 1984.

[8] P. Flajolet, X. Gourdon, and P. Dumas. Mellin transforms and asymptotics: harmonic sums. Theoret. Comput. Sci., 144(1-2):3-58, 1995. Special volume on mathematical analysis of algorithms.

[9] P. Flajolet, P. Grabner, P. Kirschenhofer, H. Prodinger, and R. F. Tichy. Mellin transforms and asymptotics: digital sums. Theoret. Comput. Sci., 123(2):291-314, 1994.

[10] A. O. Gel'fond. Sur les nombres qui ont des propriétés additives et multiplicatives données. Acta Arith., 13:259-265, 1967/1968.

[11] W. M. Y. Goh and E. Schmutz. The number of distinct part sizes in a random integer partition. J. Combin. Theory Ser. A, 69(1):149-158, 1995.

[12] H.-K. Hwang. Limit theorems for the number of summands in integer partitions. J. Combin. Theory Ser. A, 96(1):89-126, 2001.

[13] A. E. Ingham. A Tauberian theorem for partitions. Ann. of Math. (2), 42:1075-1090, 1941.

[14] C. Mauduit and A. Sárközy. On the arithmetic structure of sets characterized by sum of digits properties. $J$. Number Theory, 61(1):25-38, 1996.

[15] G. Meinardus. Asymptotische Aussagen über Partitionen. Math. Z., 59:388-398, 1954.

[16] K. F. Roth and G. Szekeres. Some asymptotic formulae in the theory of partitions. Quart. J. Math., Oxford Ser. (2), 5:241-259, 1954.

[17] E. Schmutz. Part sizes of random integer partitions. Indian J. Pure Appl. Math., 25(6):567-575, 1994.

[18] N. J. A. Sloane. The On-Line Encyclopedia of Integer Sequences. Published electronically at http://www.research.att.com/ njas/sequences.

[19] G. Tenenbaum. Introduction à la théorie analytique et probabiliste des nombres, volume 1 of Cours Spécialisés [Specialized Courses]. Société Mathématique de France, Paris, second edition, 1995.

[20] J. M. Thuswaldner and R. F. Tichy. Waring's problem with digital restrictions. Israel J. Math., 149:317-344, 2005. Probability in mathematics. 
Manfred Madritsch, Institute of Statistics, Graz University of Technology, Steyrergasse 30/II, 8010 Graz Austria

E-mail address: madritsch@finanz.math.tugraz.at

Stephan Wagner, Department of Mathematical Sciences, Stellenbosch University, Private Bag X1, Matieland 7602, South Africa

E-mail address: swagner@sun.ac.za 